\title{
Concurrent ruptured endometrioma with appendiceal endometriosis: a case report
}

\author{
Priyanka Pipara*, Ramna Banerjee
}

Department of Obstetrics and Gynecology, Apollo Gleneagles Hospital, Kolkata, West Bengal, India

Received: 26 March 2017

Accepted: 26 April 2017

\section{*Correspondence:}

Dr. Priyanka Pipara,

E-mail: priyankajain3107@gmail.com

Copyright: (c) the author(s), publisher and licensee Medip Academy. This is an open-access article distributed under the terms of the Creative Commons Attribution Non-Commercial License, which permits unrestricted non-commercial use, distribution, and reproduction in any medium, provided the original work is properly cited.

\begin{abstract}
Endometriosis is an oestrogen dependent inflammatory disease characterised by presence of endometrial tissue outside the uterine cavity. It affects $15 \%$ of female patients in reproductive age. Endometriosis is a very common cause of chronic pelvic pain and subfertility in females. We present a case of a 26-year-old woman with chronic lower abdominal pain on medical management of endometriosis. She presented to us with acute abdominal pain and underwent diagnostic laparoscopy. During surgery, we observed minimal haemoperitoneum with frozen pelvis. The appendix appeared slightly inflamed and an appendicectomy with adhesiolysis was done. The histopathological examination showed endometriosis of appendix. Her postoperative period was uneventful. The patient has been followed up postoperatively and is currently doing well.
\end{abstract}

Keywords: Abdominal pain, Appendix, Endometriosis, Laparoscopy

\section{INTRODUCTION}

Endometriosis is an oestrogen dependent inflammatory disease characterised by presence of endometrial tissue outside the uterine cavity. ${ }^{1}$ It affects $15 \%$ of female patients in reproductive age. ${ }^{2}$ Endometriosis is a very common cause of chronic pelvic pain and subfertility in females. Ectopic endometrial tissue is most commonly found in ovaries, peritoneum, uterosacral ligaments and posterior cul-de-sac. It affects gastrointestinal tract in around $12 \%$ of cases but appendiceal endometriosis is uncommon, occurs only in $3 \%$ cases of gastrointestinal endometriosis. ${ }^{3}$

Endometriosis of appendix may be asymptomatic or present as acute or chronic abdominal pain. In these cases, preoperative diagnosis is difficult and intraoperative inspection may not show any evidence of endometriosis. Laparoscopy is the gold standard for the diagnosis of endometriosis. Definitive diagnosis is only made after the histopathological examination. ${ }^{4}$ Herein we report a rare case of concurrent ruptured ovarian endometrioma and appendiceal endometriosis in a woman with acute abdominal pain.

\section{CASE REPORT}

A 26-year female patient, P1L1 first presented to us one year back with a complaint of painful periods as well as lower abdominal pain throughout the month. She had a transvaginal sonography done that suggested a small endometriotic cyst.

She was started on GnRH agonists and treatment was continued for 6 months, during this time she became amenorrheic and completely pain free. Subsequently monthly oral contraceptive pills were given. She remained well for 1 year then she presented again with painful periods and lower abdominal discomfort. The pain was mainly on the right side. A transvaginal sonography was done that showed a $2.0 \mathrm{~cm} \times 3.0 \mathrm{~cm}$ small right ovarian cyst, possibly haemhorragic. She was given 
another dose of GNRH analogue with addback therapy and analgesia with the aim to suppress symptoms. However a week later she presented in emergency with acute abdominal pain. On admission her vitals were stable, abdominal examination showed deep tenderness in lower abdomen and no guarding, rigidity and palpable mass. All blood investigations were normal. She had a CECT done that suggested bilateral bulky ovaries with mildly dilated appendix consistent with acute appendicitis.

She underwent diagnostic laparoscopy which revealed about $50 \mathrm{ml}$ of haemoperitoneum with frozen pelvis. Dense adhesions between omentum and anterior abdominal wall with rectosigmoid adherent to posterior surface of uterus and the ovaries were buried deep down in adhesions. No obvious ovarian cyst was identified. The appendix appeared slightly inflamed and an appendicectomy with adhesiolysis was done by surgeon. A levonorgestrel releasing intrauterine device was also inserted at the end of the procedure. The histopathological examination of appendix showed endometriosis (Figure 1). Her postoperative period was uneventful. The patient has been followed up postoperatively and is currently doing well.

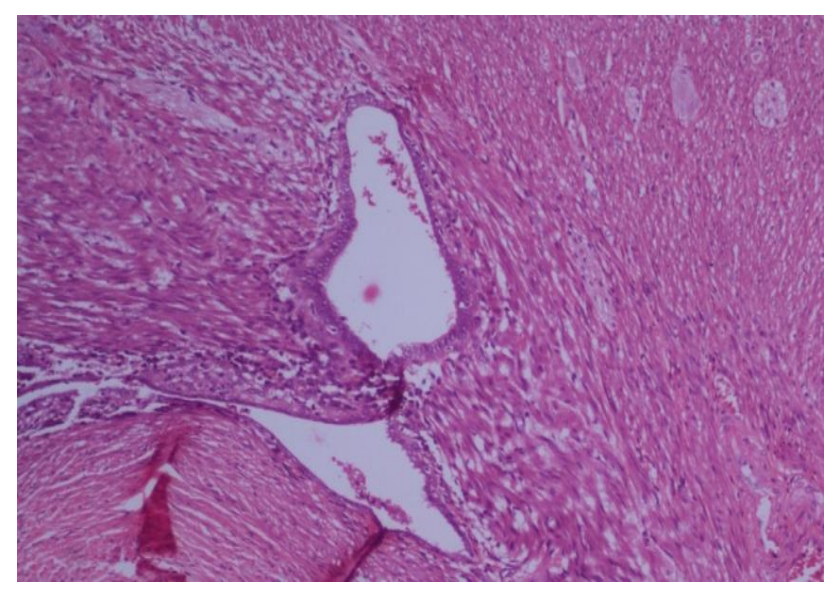

Figure 1: Microscopic picture of appendix endometrial glands surrounded by endometrial stroma.

\section{DISCUSSION}

Endometriosis is presence of endometrial tissue outside the uterine cavity and its musculature. It is commonly found in genital organs and pelvic peritoneum. ${ }^{5}$ Endometriosis present outside the genital tract is called extragenital endometriosis and it can affect GIT, lung, bladder, heart and surgical scars. Literature shows that it can present in any organ and tissue in the body. Spleen is the only site where endometriosis is not seen. ${ }^{6}$ Reported incidence of appendiceal endometriosis is between 0.054 to $0.8 \% .^{7,8}$ Various theories have been described to explain pathogenesis of extragenital endometriosis like retrograde menstruation theory, coelomic metaplasia theory, induction theory and recently developed cellular immunity theory. ${ }^{6}$ These patients can present with chronic abdominal pain or acute abdominal pain and rarely with symptoms of acute appendicitis, abdominal colic, melena and lower gastrointestinal bleeding but most of the cases are asymptomatic. ${ }^{9}$ Inflammation of appendix can occur due to occlusion of lumen by endometrioma or hemorrhage within the seromuscular layer of appendix.

Laboratory tests are of limited value for the diagnosis of appendiceal endometriosis. Computed tomography may show evidence of acute appendicitis but findings are usually nonspecific in cases of endometriosis as seen in our case. ${ }^{1}$ Laparoscopy is considered gold standard for diagnosis of endometriosis. In our patient during laparoscopy gross inspection of appendix did not show any evidence of endometriosis but microscopic examination showed features of endometriosis. Our patient had history of chronic abdominal pain which was not related to her menstruation. Endometriosis of appendix was never suspected preoperatively. Many patients with appendiceal endometriosis experience chronic pelvic pain which affects quality of life and symptoms resolution is noticed after appendectomy as seen in our case. ${ }^{10}$

Appendectomy during surgical treatment of pelvic endometriosis is controversial but can be considered after informing patient and relatives about the possible contribution of appendiceal endometriosis to the pelvic discomfort regardless of concurrent symptoms or gross finding of the appendix. ${ }^{11}$

\section{CONCLUSION}

In summary endometriosis of appendix is very rare and preoperative diagnosis is difficult. It can present along with pelvic endometriosis and should be considered in differential diagnosis of women of childbearing age complaining of nonspecific recurrent lower abdominal pain.

Funding: No funding sources

Conflict of interest: None declared

Ethical approval: Not required

\section{REFERENCES}

1. Yoon J, Lee YS, Chang H-S, Park CS. Endometriosis of the appendix. Ann Surg Treat Res. 2014;87(3):144-7.

2. Khairy GA. Endometriosis of the appendix: a trap for the unwary. Saudi J Gastroenterol Off J Saudi Gastroenterol Assoc. 2005;11(1):45-7.

3. Hasegawa T, Yoshida K, Matsui K. Endometriosis of the appendix resulting in perforated appendicitis. Case Rep Gastroenterol. 2007;1(1):27-31.

4. Al Oulaqi NS, Hefny AF, Joshi S, Salim K, AbuZidan FM. Endometriosis of the appendix. Afr Health Sci. 2008;8(3):196-8. 
5. Uncu H, Taner D. Appendiceal endometriosis: two case reports. Arch Gynecol Obstet. 2008;278(3):2735.

6. Papavramidis TS, Sapalidis K, Michalopoulos N, Karayanopoulou G, Raptou G, Tzioufa V, et al. Spontaneous abdominal wall endometriosis: a case report. Acta Chir Belg. 2009;109(6):778-81.

7. Collins DC. A study of 50,000 specimens of the human vermiform appendix. Surg Gynecol Obstet. 1955;101(4):437-45.

8. Berker B, Lashay N, Davarpanah R, Marziali M, Nezhat CH, Nezhat C. Laparoscopic appendectomy in patients with endometriosis. J Minim Invasive Gynecol. 2005;12(3):206-9.

9. Laskou S, Papavramidis TS, Cheva A, Michalopoulos N, Koulouris C, Kesisoglou I, et al.
Acute appendicitis caused by endometriosis: a case report. J Med Case Reports. 2011;5:144.

10. Barrier BF, Frazier SR, Brennaman LM, Taylor JC, Ramshaw BJ. Catamenial appendicitis. Obstet Gynecol. 2008;111(2 Pt 2):558-61.

11. Wie HJ, Lee JH, Kyung MS, Jung US, Choi JS. Is incidental appendectomy necessary in women with ovarian endometrioma? Aust N Z J Obstet Gynaecol. 2008;48(1):107-11.

Cite this article as: Pipara $\mathrm{P}$, Banerjee $\mathrm{R}$.

Concurrent ruptured endometrioma with appendiceal endometriosis: a case report. Int J Reprod Contracept Obstet Gynecol 2017;6:2611-3. 